\title{
Can blood urea Nitrogen-to-Albumin ratio predict mortality in patients with moderate-to-severe COVID-19 pneumonia hospitalized in the intensive care unit?
}

\author{
Filiz Ata ${ }^{\top} \odot$, Ahmet Kagan $\mathrm{As}^{2} \odot$, Mesut Engin ${ }^{2} \odot$, \\ Nurcan Kacmaz Kat ${ }^{3} \odot$, Yusuf Ata $^{2} \odot$, Tamer Turk ${ }^{2 *} \odot$
}

SUMMARY

OBJECTIVE: Many laboratory parameters allow to follow up the course of the disease and reveal its clinical severity, particularly in patients with coronavirus disease 2019 (COVID-19) pneumonia. In this study, we aimed to investigate the role of the blood urea nitrogen-to-albumin ratio in predicting the mortality in COVID-19 patients with moderate-to-severe disease who are hospitalized in the intensive care unit. METHODS: A total of 358 patients who were hospitalized in intensive care unit at our hospital between November 1, 2020 and May 15, 2021 were included in this study. During their course of intensive care, surviving patients were included in Group 1 and nonsurviving patients in Group 2. RESULTS: There were no statistically significant differences between the two groups in terms of gender, smoking, and chronic obstructive pulmonary disease rates. In multivariate logistic regression analysis, advanced age (OR 1.038, 95\% CI 1.014-1.064, p=0.002), neutrophilto-lymphocyte ratio (OR $1.226,95 \% \mathrm{Cl} 1.020-1.475, \mathrm{p}=0.030)$, blood urea nitrogen-to-albumin ratio $(\mathrm{OR} 2.693,95 \% \mathrm{Cl} 2.019-3.593$, $\mathrm{p}<0.001$ ), and chest computed tomography severity score (OR $1.163,95 \% \mathrm{Cl} 1.105-1.225, \mathrm{p}<0.001)$ values were determined as independent predictors for in-hospital mortality.

CONCLUSION: In this study, we showed that the blood urea nitrogen-to-albumin ratio, which was previously shown as a predictor of mortality in patients with various pneumonia, was an independent predictor of mortality in patients with COVID-19 pneumonia.

KEYWORDS: COVID-19. Pandemic. Inflammation. Mortality. Intensive care.

\section{INTRODUCTION}

Besides respiratory and gastrointestinal problems, coronaviruses may also cause neurological and visceral organ damage. The coronavirus disease 2019 (COVID-19), which emerged in Wuhan, China, at the end of 2019, caused a pandemic ${ }^{1}$. Due to this, millions of people lost their lives, and the entire world is affected for more than a year ${ }^{2}$.

With the COVID-19 pandemic, it has become especially important to predict morbidity and mortality in patients.
Treatment of vulnerable groups, such as those with moderate and severe disease, will play a key role in the management of the "health crisis" caused by the pandemic. Many laboratory parameters, such as C-reactive protein, fibrinogen, troponin, ferritin, and DD-dimer, allow to follow up the course of the disease and reveal its clinical severity, particularly in patients with COVID-19 pneumonia ${ }^{3}$. One of the most important of these is albumin. Synthesized by the liver, it plays an important

\footnotetext{
'University of Health, Bursa Yuksek Ihtisas Training and Research Hospital Sciences, Department of Anesthesiology and Reanimation - Bursa, Turkey. ${ }^{2}$ University of Health Sciences, Bursa Yuksek Ihtisas Training and Research Hospital, Department of Cardiovascular Surgery - Bursa, Turkey. ${ }^{3}$ University of Health Sciences, Bursa Yuksek Ihtisas Training and Research Hospital, Department of Radiology - Bursa, Turkey.

*Corresponding author: tturkon@hotmail.com

Conflicts of interest: the authors declare there are no conflicts of interest. Funding: none.

Received on June 28, 2021. Accepted on August 15, 2021.
} 
role in maintaining the osmotic pressure as well as in the transport of many vital substances ${ }^{4}$. In a study, it was shown that it may be a predictor of mortality in hospitalized COVID-19 patients 5 . In addition, the amount of urea absorbed by the kidneys causes an increase in blood urea nitrogen (BUN) levels. This can also show dehydration, a common condition among patients with pneumonia, and indicate a poor prognosis ${ }^{6}$. In the light of this information, the ratio of blood urea nitrogen-to-albumin $(\mathrm{B} / \mathrm{A})$ appears to be an important prognostic marker. In a recent study, it was shown that it may be a predictor of mortality in patients with aspiration pneumonia ${ }^{7}$.

In this study, we aimed to investigate the role of the $\mathrm{B} / \mathrm{A}$ ratio in predicting mortality in COVID-19 patients with moderate-to-severe disease who are hospitalized in the intensive care unit (ICU).

\section{METHODS}

Patients with moderate and severe COVID-19 pneumonia hospitalized in the ICU between November 1, 2020 and May 15, 2021 were consecutively included in this study. The real-time polymerase chain reaction (RT-PCR) tests of nasal and pharyngeal swab samples of all patients included in the study were found positive. Demographic data of all patients (e.g., age, the presence of hypertension, diabetes mellitus, coronary artery disease) and laboratory parameters at the time of admission were noted. Intubated patients admitted to the ICU and patients with critical disease, malignancy, known systemic inflammatory disease, liver failure, and serum creatinine values above 2 $\mathrm{mg} / \mathrm{dL}$ were excluded from the study. Involvement rates were calculated by evaluating the thorax tomography images of all patients at the time of admission. During their course of intensive care, surviving patients were included in Group 1 and nonsurviving patients in Group 2.

Indications for hospitalization in the ICU of all patients were in line with the recommendations of the scientific committee of our country ${ }^{8}$. Chest Computed Tomography Severity Score (CT-SS) scoring, which was developed by Yang et al. ${ }^{\text {, }}$ was utilized to assess chest $\mathrm{CT}$ images.

\section{Statistical analysis}

In this study, SPSS version 21.0 (IBM Statistical Package for the Social Sciences Statistic Inc., Chicago, IL, USA) program was utilized to analyze the data. "Kolmogorov-Smirnov test and Shapiro-Wilk test" were used for normality distribution analysis. Student's $t$ test was used for the data presenting normal distribution and Mann-Whitney $U$ test for those that did not conform to normal distribution. These data were shown as mean \pm standard deviation or as mean (interquartile range, 25th percentile-75th percentile). Categorical variables were shown as frequency and percentage, and "chi-square test" was used for analysis. Multivariate binary logistic regression analysis was utilized to analyze mortality predictors. A p $<0.05$ was accepted statistically significant. In predicting in-hospital mortality, receiver operating characteristics (ROC) curve analysis was performed in order to calculate neutrophil-to-lymphocyte ratio (NLR), CT-SS, B/A ratio, and area under the curves (AUCs). Spearman correlation analysis was utilized to assess a possible linear association between B/A ratio and CT-SS.

\section{RESULTS}

A total of 358 patients were included in the study. Those who did not develop in-hospital mortality were included in Group $1(\mathrm{n}=209$, median age $=48$ [39-61.5] years $)$ and those who did were in Group 2 ( $n=149$, median age $=66$ [50.5-77] years). There were no statistically significant differences between the two groups in terms of gender, smoking, and chronic obstructive pulmonary disease rates. Age, diabetes mellitus, hypertension, and coronary artery disease rates were significantly higher in Group 2 compared to Group 1 ( $\mathrm{p}<0.001, \mathrm{p}=0.002$, $\mathrm{p}=0.030$, and $\mathrm{p}<0.001$, respectively). Also admission CT-SS of the patients was higher in group $2(\mathrm{p}<0.001)$. Demographic characteristics of all patients are presented in Table 1.

Preoperative blood values of the patients are provided in Table 1. The two Groups were similar in terms of white blood cell and platelet values. In Group 2, while neutrophil counts, NLR, ferritin, troponin I, D-dimer, fibrinogen, C-reactive protein, blood urea nitrogen, creatinine, and $\mathrm{B} / \mathrm{A}$ ratio values were significantly higher $(\mathrm{p}<0.001, \mathrm{p}<0.001, \mathrm{p}<0.001$, $\mathrm{p}<0.001, \mathrm{p}=0.001, \mathrm{p}=0.006, \mathrm{p}<0.001, \mathrm{p}<0.001, \mathrm{p}<0.001$, and $\mathrm{p}<0.001$ respectively), hemoglobin, lymphocyte, and albumin values were significantly lower $(\mathrm{p}=0.009, \mathrm{p}<0.001$, and $\mathrm{p}<0.001$, respectively).

Multivariate logistic regression analysis was performed to evaluate the predictive value of certain parameters in terms of in-hospital mortality. In this analysis; advanced age (OR 1.038, 95\%CI 1.014-1.064, $\mathrm{p}=0.002$ ), NLR (OR 1.226, 95\%CI $1.020-1.475, \mathrm{p}=0.030$ ), B/A ratio (OR 2.693, 95\%CI 2.0193.593, $\mathrm{p}<0.001$ ), and CT-SS (OR 1.163, 95\%CI 1.105-1.225, $\mathrm{p}<0.001)$ values were determined as independent predictors for in-hospital mortality (Table 2).

ROC curve analysis was performed to evaluate $\mathrm{B} / \mathrm{A}$ ratio, NLR, and CT-SS in predicting mortality. The cutoff value of $\mathrm{B} / \mathrm{A}$ ratio was 3.4 ( AUC $0.823,95 \% \mathrm{CI} 0.777-0.870$, $\mathrm{p}<0.001$, with $74.5 \%$ sensitivity and $75.6 \%$ specificity) and that of NLR was 2.73 (AUC 0.749, 95\%CI 0.696-0.802, $\mathrm{p}<0.001$, with $68.5 \%$ sensitivity and $70.8 \%$ specificity) and CT-SS was 13.5 
(AUC $0.754,95 \%$ CI $0.702-0.805$, $\mathrm{p}<0.001$, with $63.8 \%$ sensitivity and $77.5 \%$ specificity) (Figure 1 ).

There was a mild positive correlation between $\mathrm{B} / \mathrm{A}$ ratio and CT-SS $(\mathrm{r}=0.230, \mathrm{p}<0.001)$.

\section{DISCUSSION}

In this study, we showed that the $\mathrm{B} / \mathrm{A}$ ratio is an independent predictor of mortality in patients with moderate-to-severe COVID-19 pneumonia hospitalized in the ICU. In addition, we found that CT-SS, advanced age, and NLR values were independent predictors of mortality. There was also a mild correlation between CT-SS and B/A ratio.

Serum albumin is an acute-phase reactant with antioxidant properties. It plays a significant role in the destruction of free oxygen radicals synthesized during oxidative stress ${ }^{10,11}$. COVID-19 disease also induces an oxidative stress state in humans, and a study showed that low albumin can predict the severity of the disease ${ }^{12}$. In another study conducted on 319 hospitalized COVID-19 patients, Violi et al. ${ }^{5}$ investigated the effect of albumin on mortality. The authors proposed the idea that albumin levels could be used to distinguish COVID-19 patients with elevated mortality risk ${ }^{5}$. Li et al. investigated the effect of albumin on clinical outcomes in 134 COVID-19 patients and found low albumin levels to be significantly associated with pneumonia severity as well as mortality in patients with critical disease ${ }^{13}$. In our study, albumin values were significantly lower in nonsurviving patients.

BUN value is an important indicator of dehydration status and is known to be associated with poor clinical outcomes

Table 1. Demographic features and admission clinic data and laboratory values of the patients.

\begin{tabular}{|c|c|c|c|}
\hline & $\begin{array}{c}\text { Group } 1 \\
n=209 \\
\text { (Survivors) }\end{array}$ & $\begin{array}{c}\text { Group } 2 \\
n=149 \\
\text { (Nonsurvivors) }\end{array}$ & p-value \\
\hline Age & $48(39-61.5)$ & $66(50.5-77)$ & $<0.001^{b}$ \\
\hline Male/female gender & $82 / 127$ & $66 / 83$ & $0.338^{\mathrm{a}}$ \\
\hline Smoking, n (\%) & $52(24.9)$ & $50(33.6)$ & $0.073^{a}$ \\
\hline Hypertension, n (\%) & $56(26.8)$ & $56(37.6)$ & $0.030^{\mathrm{a}}$ \\
\hline Coronary artery disease, n (\%) & $23(11)$ & $39(26.2)$ & $<0.001^{a}$ \\
\hline Diabetes mellitus, n (\%) & $33(15.8)$ & $44(29.5)$ & $0.002^{\mathrm{a}}$ \\
\hline COPD, n (\%) & $13(6.2)$ & $14(9.4)$ & $0.262^{\mathrm{a}}$ \\
\hline CT-SS & $11(9-13)$ & $17(12-22)$ & $<0.001^{b}$ \\
\hline WBC $\left(10^{3} / \mathrm{mm}^{3}\right)$ & $5.89(4.66-7.28)$ & $6.09(4.66-8.47)$ & $0.088^{b}$ \\
\hline Hemoglobin (g/dL) & 13.1(11.8-14.1) & $12.6(11.2-13.7)$ & $0.009^{b}$ \\
\hline Platelet $\left(10^{3} / \mathrm{mm}^{3}\right)$ & $215(173-253)$ & $224(164.5-316.5)$ & $0.085^{b}$ \\
\hline Neutrophil $\left(10^{3} / \mathrm{mm}^{3}\right)$ & $3.4(2.5-4.4)$ & $4.3(3.2-6.2)$ & $<0.001^{b}$ \\
\hline Lymphocyte $\left(10^{3} / \mathrm{mm}^{3}\right)$ & $1.7(1.2-2.2)$ & $1.1(0.8-1.4)$ & $<0.001^{b}$ \\
\hline Neutrophil-to-lymphocyte ratio & $2.1(1.3-2.9)$ & $3.7(2.3-6.9)$ & $<0.001^{b}$ \\
\hline Ferritin (mg/L) & $144(70-295.2)$ & $275(135-433.4)$ & $<0.001^{b}$ \\
\hline Troponin I (mg/L) & $3.14(1-7.49)$ & $5(3-13)$ & $<0.001^{b}$ \\
\hline D-Dimer (ng/mL) & $0.53(0.3-1.04)$ & $0.81(0.53-1.36)$ & $0.001^{b}$ \\
\hline Fibrinogen (mg/dL) & $415(310.5-550)$ & $508(357.5-650)$ & $0.006^{b}$ \\
\hline Albumin (g/dL) & $3.9(3.6-4.2)$ & $3.75(3.5-4)$ & $<0.001^{b}$ \\
\hline C-reactive protein (mg/L) & $11.3(3.4-49.6)$ & $40(11.6-82.6)$ & $<0.001^{b}$ \\
\hline BUN (mg/dL) & $10.8(8.9-12.6)$ & $17.8(12.5-23.1)$ & $<0.001^{b}$ \\
\hline Creatinine (mg/dL) & $0.77(0.64-0.96)$ & $1.03(0.7-1.39)$ & $<0.001^{b}$ \\
\hline B/A ratio (mg/g) & $2.77(2.23-3.39)$ & $4.69(3.38-6.42)$ & $<0.001^{\mathrm{b}}$ \\
\hline
\end{tabular}

COPD: chronic obstructive pulmonary disease; CT-SS: Computed Tomography Severity Score; WBC: white blood cell; BUN: blood urea nitrogen; B/A: BUN/albumin. ${ }^{a} \chi^{2}$ test. ${ }^{b}$ Mann-Whitney $U$ test. Data are expressed as median and interquartile range (25th-75th percentile). 
in patients with heart failure and community-acquired pneumonia ${ }^{14,15}$. The study on 337 COVID-19 patients performed by Liu et al. showed that high BUN values may be associated with mortality ${ }^{16}$. Cheng et al. investigated the importance of BUN and D-dimer values in predicting in-hospital mortality in 305 COVID-19 patients.

BUN values were significantly higher among nonsurvivors $(\mathrm{p}<0.0001)^{17}$.

In the light of this information about BUN and albumin, an increase in BUN values and a decrease in albumin values, which results in an increased B/A ratio, appear as important prognostic markers. In recent studies, B/A ratio was shown to be a mortality predictor for various diseases ${ }^{18,19}$. In a study on 175 patients with community-acquired pneumonia, Ugajin et al. ${ }^{10}$ investigated the effect of the $\mathrm{B} / \mathrm{A}$ ratio on clinical outcomes and identified the $\mathrm{B} / \mathrm{A}$ ratio as an independent predictor of ICU need (OR 1.27, 95\%CI 1.09-1.47, p=0.002) and mortality (OR 1.10, 95\%CI 1.01-1.20, p=0.037) ${ }^{10}$. In a recent study, Ryu et al. ${ }^{7}$ investigated the prognostic role of the $\mathrm{B} / \mathrm{A}$ ratio among 443 patients with aspiration pneumonia and concluded that $\mathrm{B} / \mathrm{A}>7$ was an independent predictor of 28 -day mortality (OR 3.40, 95\%CI 1.87-6.21, p<0.001). In our study, we determined that the $\mathrm{B} / \mathrm{A}$ ratio is an independent predictor of mortality in patients with COVID-19 pneumonia needing intensive care.

An increase in the neutrophil ratio and a decrease in the lymphocyte ratio, resulting in elevated NLR, play a significant role in the progression and prognosis of various diseases ${ }^{20,21}$. In addition, lymphopenia is the most common hematological finding, seen at a rate of $83 \%$ among hospitalized COVID-19 patients $^{22}$. In a meta-analysis of 38 currently published articles, including 5699 patients with severe disease and 6033 nonsurviving patients, high NLR values were shown to be associated

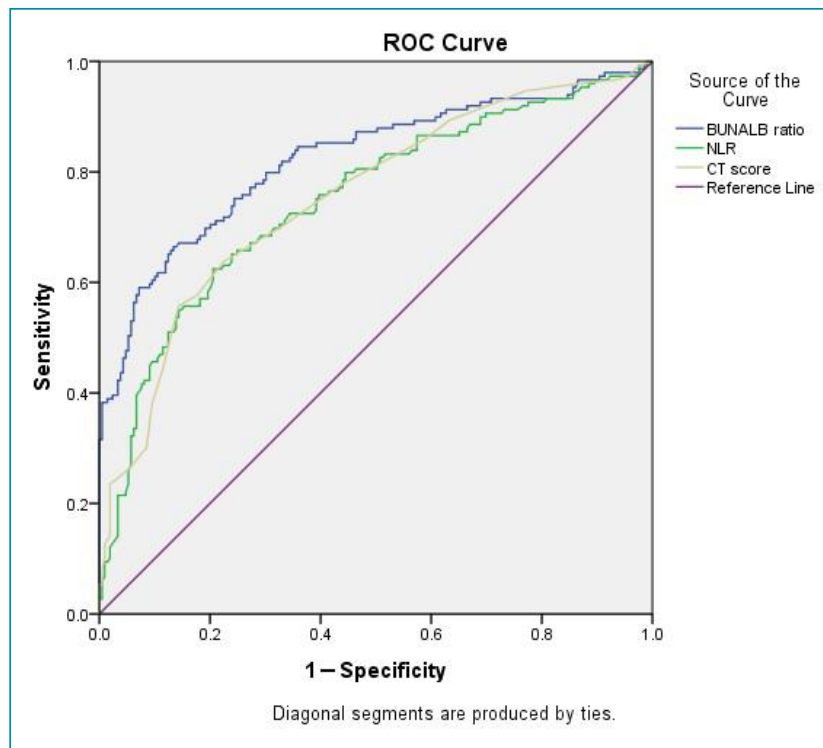

Figure 1. Data of the area under the curve, confidence interval, and cutoff values in receiver operating characteristic curve analysis for blood urea nitrogen-to-albumin ratio (cutoff 3.4, AUC 0.823, 95\% CI 0.777-0.870, p<0.001, with $74.5 \%$ sensitivity and $75.6 \%$ specificity), NLR (cutoff 2.73 , AUC 0.749, 95\% Cl 0.696-0.802, p<0.001, with 68.5\% sensitivity and $70.8 \%$ specificity) and CT-SS (cutoff 13.5 , AUC 0.754, 95\% Cl 0.702-0.805, p<0.001, with 63.8\% sensitivity and $77.5 \%$ specificity).

Table 2. Multivariate logistic regression analysis to identify factors affecting in-hospital mortality.

\begin{tabular}{l|c|c|c}
\multirow{2}{*}{ Age } & \multicolumn{3}{|c}{ Multivariate analysis } \\
\cline { 2 - 4 } & p-value & Exp(B) odds ratio & 95\%Cl Lower-Upper \\
\hline Hypertension & 0.002 & 1.038 & $1.014-1.064$ \\
\hline Diabetes mellitus & 0.142 & 1.810 & $0.820-3.995$ \\
\hline Hemoglobin & 0.307 & 0.645 & $0.279-1.494$ \\
\hline Troponin I & 0.656 & 0.962 & $0.813-1.139$ \\
\hline Creatinine & 0.070 & 0.983 & $0.964-1.001$ \\
\hline D-Dimer & 0.237 & 2.065 & $0.620-6.872$ \\
\hline Fibrinogen & 0.366 & 1.015 & $0.983-1.047$ \\
\hline C- reactive protein & 0.959 & 1.000 & $0.999-1.002$ \\
\hline Neutrophil-to-lymphocyte ratio & 0.170 & 0.995 & $0.987-1.002$ \\
\hline B/A ratio & 0.030 & 1.226 & $1.020-1.475$ \\
\hline CT-SS & $<0.001$ & 2.693 & $2.019-3.593$ \\
\hline
\end{tabular}

CT-SS: Computed Tomography Severity Score; B/A: blood urea nitrogen/albumin. 
with disease severity and mortality ${ }^{23}$. In our study, a high NLR value was an independent predictor of mortality, in line with the literature.

The most important limitation of our study is its single-center and retrospective design. In addition, the number of patients was sparse. Evaluations were made by considering the clinical parameters of the patients at the time of admission to the ICU. These dynamic parameters may change hourly or daily during patient follow-ups. Our study needs to be supported with prospective multicenter studies, including clinical follow-up parameters.

\section{CONCLUSIONS}

This is the first study to show that the B/A ratio, which was previously shown as a predictor of mortality in patients with various pneumonia, was an independent predictor of mortality in patients with COVID-19 pneumonia. In addition, unlike many mortality studies, we calculated the CT-SS values of all patients and showed a slightly positive correlation between the $\mathrm{B} / \mathrm{A}$ ratio and CT-SS.

\section{AUTHORS' CONTRIBUTIONS}

FA: Conceptualization, Data curation, Investigation, Methodology, Supervision, Validation, Visualization, Writing - original draft, Writing - review \& editing. AKA: Investigation, Methodology, Supervision, Validation, Visualization, Writingoriginal draft, Writing - review \& editing. ME: Investigation, Methodology, Supervision, Validation, Visualization, Writing original draft, Writing - review \& editing. NKK: Investigation, Methodology, Supervision, Validation, Visualization, Writing - original draft, Writing - review \& editing. YA: Supervision, Validation, Visualization, Writing - original draft, Writing review \& editing. TT: Supervision, Validation, Visualization, Writing - original draft, Writing - review \& editing.

\section{REFERENCES}

1. Ali S, Muhammad A, Mehar T, Rehman NU, Mehmood N, Iqbal W. Serological screening of suspected COVID-19 patients in Pakistan. J Coll Physicians Surg Pak. 2021;31(Supp1):S71-4. https://doi.org/10.29271/jcpsp.2021.Supp1.S71

2. Wu Z, McGoogan JM. Characteristics of and important lessons from the coronavirus disease 2019 (COVID-19) outbreak in China: summary of a report of 72314 cases from the Chinese Center for Disease Control and Prevention. JAMA. 2020;323(13):1239-42. https://doi.org/10.1001/ jama.2020.2648

3. As AK, Erdolu B, Duman B, Yazgan E, Eris C, Aydin U, et al. Can a modified-simplified pulmonary embolism severity index ( $m$-SPESI) be used to predict the need for intensive care in hospitalized COVID-19 patients? J Thromb Thrombolysis. 2021:1-7. https://doi.org/10.1007/s11239-021-02405-7

4. Bernardi M, Angeli P, Claria J, Moreau R, Gines P, Jalan R, et al. Albumin in decompensated cirrhosis: new concepts and perspectives. Gut. 2020;69(6):1127-38. https://doi. org/10.1136/gutjnl-2019-318843

5. Violi F, Cangemi R, Romiti GF, Ceccarelli G, Oliva A, Alessandri $F$, et al. Is albumin predictor of mortality in COVID-19? Antioxid Redox Signal. 2021;35(2):139-42. https://doi.org/10.1089/ ars.2020.8142

6. Feng DY, Zhou YQ, Zou XL, Zhou M, Yang HL, Chen XX, et al. Elevated blood urea nitrogen-to-serum albumin ratio as a factor that negatively affects the mortality of patients with hospital-acquired pneumonia. Can J Infect Dis Med Microbiol. 2019:1547405 https://doi.org/10.1155/2019/1547405

7. Ryu S, Oh SK, Cho SU, You Y, Park JS, Min JH, et al. Utility of the blood urea nitrogen to serum albumin ratio as a prognostic factor of mortality in aspiration pneumonia patients. Am J Emerg Med. 2021;43:175-9. https://doi.org/10.1016/j. ajem.2020.02.045
8. TC Ministry of Health. Daily COVID-19 table [internet]. Ankara: T.C. Ministry of Health; 2021. [cited on Jun. 4, 2021]. Available from: https://covid19bilgi.saglik.gov.tr

9. Yang R, Li X, Liu H, Zhen Y, Zhang X, Xiong Q, et al. Chest CT severity score: an imaging tool for assessing severe COVID-19. Radiol Cardiothorac Imaging. 2020;2(2):e200047. https://doi. org/10.1148/ryct.2020200047

10. Ugajin M, Yamaki K, Iwamura N, Yagi T, Asano T. Blood urea nitrogen to serum albumin ratio independently predicts mortality and severity of community-acquired pneumonia. Int J Gen Med. 2012;5:583-9. https://doi.org/10.2147/JJGM.S33628

11. Inoue M, Nakashima R, Enomoto M, Koike Y, Zhao X, Yip K, et al. Plasma redox imbalance caused by albumin oxidation promotes lung-predominant NETosis and pulmonary cancer metastasis. Nat Commun. 2018;9(1):5116. https://doi. org/10.1038/s41467-018-07550-x

12. Liu $Y$, Yang $Y$, Zhang $C$, Huang F, Wang F, Yuan J, et al. Clinical and biochemical indexes from 2019-nCoV infected patients linked to viral loads and lung injury. Sci China Life Sci.2020;63(3):36474. https://doi.org/10.1007/s11427-020-1643-8

13. Li J, Li M, Zheng S, Li M, Zhang M, Sun M, et al. Plasma albumin levels predict risk for nonsurvivors in critically ill patients with COVID-19. Biomark Med. 2020;14(10):827-37. https://doi. org/10.2217/bmm-2020-0254

14. Aronson D, Mittleman MA, Burger AJ. Elevated blood urea nitrogen level as a predictor of mortality in patients admitted for decompensated heart failure. Am J Med. 2004;116:46673. https://doi.org/10.1016/j.amjmed.2003.11.014

15. Ito A, Ishida T, Tokumasu $H$, Washio $Y$, Yamazaki A, Ito $Y$, et al. Prognostic factors in hospitalized community-acquired pneumonia: a retrospective study of a prospective observational cohort. BMC Pulm Med. 2017;17(1):78. https://doi.org/10.1186/ s12890-017-0424-4 
16. Liu Q, Wang Y, Zhao X, Wang L, Liu F, Wang T, et al. Diagnostic performance of a blood urea nitrogen to creatinine ratio-based nomogram for predicting in-hospital mortality in COVID-19 patients. Risk Manag Healthc Policy. 2021;14:117-28. https:// doi.org/10.2147/RMHP.S278365

17. Cheng A, Hu L, Wang Y, Huang L, Zhao L, Zhang C, et al. Diagnostic performance of initial blood urea nitrogen combined with D-dimer levels for predicting in-hospital mortality in COVID-19 patients. Int J Antimicrob Agents. 2020;56(3):106110. https://doi.org/10.1016/j.ijantimicag.2020.106110

18. Zou XL, Feng DY, Wu WB, Yang HL, Zhang TT. Blood urea nitrogen to serum albumin ratio independently predicts 30-day mortality and severity in patients with Escherichia coli bacteraemia. Med Clin (Barc). 2021;157(5):219-25. https:// doi.org/10.1016/j.medcli.2020.06.060

19. Akahane J, Ushiki A, Kosaka M, Ikuyama Y, Matsuo A, Hachiya $T$, et al. Blood urea nitrogen-to-serum albumin ratio and a-DROP are useful in assessing the severity of pneumocystis pneumonia in patients without human immunodeficiency virus infection. J Infect Chemother. 2021;27(5):707-14. https://doi. org/10.1016/j.jiac.2020.12.017
20. Paliogiannis $P$, Fois $A G$, Sotgia $S$, Mangoni AA, Zinellu $E$, Pirina $P$, et al. Neutrophil to lymphocyte ratio and clinical outcomes in COPD: recent evidence and future perspectives. Eur Respir Rev. 2018;27(147):170113. https:// doi.org/10.1183/16000617.0113-2017

21. Abanoz M, Engin $M$. The effect of the relationship between post-cardiotomy neutrophil/lymphocyte ratio and platelet counts on early major adverse events after isolated coronary artery bypass grafting. Turk Gogus Kalp Damar Cerrahisi Derg. 2021;29(1):36-44. https://doi.org/10.5606/tgkdc. dergisi.2021.20873

22. Guan WJ, Ni ZY, Hu Y, Liang WH, Ou CQ, He JX, et al; China medical treatment expert group for Covid-19. Clinical characteristics of coronavirus disease 2019 in China. N Engl J Med. 2020;382(18):1708-20. https://doi.org/10.1056/ NEJMoa2002032

23. Simadibrata DM, Calvin J, Wijaya AD, Ibrahim NAA. Neutrophilto-lymphocyte ratio on admission to predict the severity and mortality of COVID-19 patients: a meta-analysis. Am J Emerg Med. 2021;42:60-9. https://doi.org/10.1016/j. ajem.2021.01.006 